Article

\title{
Dietary Antioxidants in Coffee Leaves: Impact of Botanical Origin and Maturity on Chlorogenic Acids and Xanthones
}

\author{
Ângelo Monteiro ${ }^{1}$ *, Silvia Colomban ${ }^{2}$, Helena G. Azinheira ${ }^{3,4}$, Leonor Guerra-Guimarães ${ }^{3,4}$, \\ Maria Do Céu Silva ${ }^{3,4}$, Luciano Navarini ${ }^{2}$ and Marina Resmini ${ }^{1 \text {,* }}$ \\ 1 Department of Chemistry, Queen Mary University of London, Mile End Road, London E1 4NS, UK \\ 2 Illycaffè S.p.A., via Flavia 143, 34100 Trieste, Italy; silvia.colomban@illy.com (S.C.); \\ luciano.navarini@illy.com (L.N.) \\ 3 Centro de Investigação das Ferrugens do Cafeeiro, Instituto Superior de Agronomia, \\ Universidade de Lisboa, 2784-505 Oeiras, Portugal; hgazinheira@isa.ulisboa.pt (H.G.A.); \\ leonorguimaraes@edu.ulisboa.pt (L.G.-G.); mariaceusilva@isa.ulisboa.pt (M.D.C.S.) \\ 4 Linking Landscape, Environment, Agricultural and Food, Instituto Superior de Agronomia, \\ Universidade de Lisboa, 1349-017 Lisboa, Portugal \\ * Correspondence: a.f.monteiro@qmul.ac.uk (Â.M.); m.resmini@qmul.ac.uk (M.R.)
}

Received: 20 November 2019; Accepted: 18 December 2019; Published: 20 December 2019

check for updates

\begin{abstract}
Natural polyphenols are important dietary antioxidants that significantly benefit human health. Coffee and tea have been shown to largely contribute to the dietary intake of these antioxidants in several populations. More recently, the use of coffee leaves to produce tea has become a potential commercial target, therefore prompting studies on the quantification of polyphenols in coffee leaves. In this study a variety of coffee leaf species, at different development stages, were analyzed using ultra-high pressure liquid chromatography. The results demonstrate that both the botanical origin of the samples and their maturity influence significantly the concentration of the antioxidants; for total chlorogenic acids a two-fold difference was found between different species and up to a three-fold variation was observed between young and mature leaves. Furthermore, the range of concentrations of chlorogenic acids in young leaves (35.7-80.8 $\mathrm{mg} / \mathrm{g}$ of dry matter) were found to be comparable to the one reported for green coffee beans. The results provide important data from which potential new commercial products can be developed.
\end{abstract}

Keywords: antioxidants; alkaloids; chlorogenic acids; coffee leaves; leaf development stages; mangiferin; xanthones

\section{Introduction}

Natural polyphenols, one of the most important groups of dietary antioxidants, include a wide variety of chemical compounds, such as flavones, flavonols, isoflavones, and phenolic acids, including chlorogenic acids (CGAs) [1]. All these secondary metabolites are widely found in fruits, vegetables, grains, and cocoa, as well as coffee, tea, and wine [2]. These phytochemicals are of great interest to nutritionists, food scientists and consumers due to their demonstrated impact on human health, especially in the prevention of degenerative and cardiovascular diseases, and also metabolic disorders [3]. Coffee and tea, in particular, have been found to play a key role in the polyphenol dietary intake of consumers, with the consumption of these drinks responsible in some cases for $>50 \%$ of polyphenols intake [4-6].

Coffee, with a global production of 158.6 million 60-kg bags in 2017 and an export value of 32.7 billion dollars [7], is the second most popular beverage worldwide after water. The chemical 
composition of the beans of the two most commercially exploited coffee species, Coffea arabica Linn. and Coffea canephora Pierre ex A. Froehner, has been extensively studied because of their relevance to cup quality, product traceability, authenticity, and safety. Significant research was carried out to understand the chemistry of green coffee beans with the aim of improving quality [8]. Coffee beans are not the only source of antioxidants in the coffee plant.

Coffee leaves have been commonly used to make infusions in many coffee producing countries such as Ethiopia, South Sudan, and Indonesia. The beverage is believed to help on the treatment of several disorders, for instance anemia, intestinal pain, and fever [9]. In 2013, Wize Monkey, a Canadian company, started selling coffee leaf tea all over the world, with great success, confirming the growing interest in leaves as a commodity. For this reason, the study of the chemical composition of coffee leaves, from which tea is made, is now a priority. A number of studies have been done to identify bioactive compounds present in coffee leaves [10-13]. The results identified three main classes of bioactive compounds, namely alkaloids, chlorogenic acids and more recently xanthones [13]. However, with one exception [11] there is a significant lack of data in literature regarding the effect of aging and interspecific variation on the antioxidants' concentration.

Chlorogenic acids (CGAs) are the main class of polyphenols in coffee beans, where they can be found in large quantities [14]. Metabolic fate and bioavailability of these compounds have been recently investigated, and were found to be absorbed and excreted to a much greater extent than many other dietary flavonoids and phenolic compounds [15]. The high accumulation of CGAs in the coffee leaves of different species has been previously reported, in which 5-caffeoylquinic acid (5-CQA) and 3,5-dicaffeoylquinic acid (3,5-DiCQA) were found to be the most abundant chlorogenic acids $[11,13,16,17]$.

Xanthones, which are famously known by mangostin and mangiferin, possess valuable biological activities with a great potential for pharmacological applications [18]. In particular, mangiferin can be mainly found in different tissues of Mangifera indica (mango tree) and Cyclopia genistoides (honeybush), which are widely used in traditional medicine [19]. Coffee leaves have been found to contain an amount of mangiferin comparable to $M$. indica leaves [20]. Recently, the accumulation of mangiferin has been demonstrated in the leaves of several coffee species, suggesting that this maybe an excellent natural source of this xanthone $[11,12]$.

The third class of bioactive compounds present in coffee leaves is alkaloids, particularly xanthines and trigonelline, whose biological properties have been studied [21,22]. Caffeine, the most widely consumed psychostimulant worldwide, exerts a wide range of effects on the human body and for this reason metabolism and pharmacokinetics have been extensively studied in the past and revised recently [21]. Caffeine and its analogues may also contribute to the overall antioxidant properties of coffee $[23,24]$. The presence of caffeine in coffee leaves makes the coffee leaf tea an alternative to the traditional tea beverage, in terms of central nervous system stimulation [10], in addition to other bioactive flavonoids including rutin, quercetin and kaempferol glycosides which have been recently reported [13].

The potential health benefits of coffee-leaf tea as beverage $[25,26]$ is of great interest to the coffee community, as a mean of adding value to waste biomass and also to coffee species with very low beans yield and, such as Coffea racemosa Lour. and Coffea eugenioides S. Moore. This work focused on the profiling and comparison of several coffee leaf species at different development stages, with the quantification of the major bioactive compounds, i.e., alkaloids, chlorogenic acids and xanthones, done by ultra-high-performance liquid chromatography (UHPLC). The results are expected to provide a phytochemical profile that will aid further applications of this source of active compounds in the food industry. 


\section{Materials and Methods}

\subsection{Chemicals}

For quantitative purposes primary reference standards were used where possible: 3-caffeoylquinic acid (3CQA), 4-caffeoylquinic acid (4CQA), 5-caffeoylquinic acid (5CQA), 3,4-dicaffeoylquinic acid (3,4-diCQA), 3,5-dicaffeoylquinic acid (3,5-diCQA), and 4,5-dicaffeoylquinic acid (4,5-diCQA) were obtained from PhytoLab GmbH \& Co. KG (Vestenbergsgreuth, Germany); caffeine (Ph. Eur. Grade), theobromine, mangiferin trigonelline hydrochloride (analytical grade) methanol and ethanol (HPLC grade) from Sigma-Aldrich Chemie $\mathrm{GmbH}$ (Steinheim, Germany). Qualitative identification was performed using synthetized standards of FQAs and pCoQAs, not commercially-available. Sodium metabisulfite was obtained from VWR International (Fountenay-sous-Boiscedex, France). All solutions were made with milliQwater system (Millipore, Molsheim, France) and methanol (7:3 v:v).

\subsection{Taxon Sampling and Plant Material}

The species, accession and origin of the coffee plants used are indicated in Table S1. Leaves were collected from plants of $C$. arabica cv. Bourbon maintained in greenhouse conditions (private collection, Illycaffè, Trieste, Italy) during the month of July 2017. A selection was made between young leaves (fully expanded leaves of the terminal node) and mature leaves (fully expanded leaves of 2nd pair from branch apex), medium sizes are reported in Table S1. C. canephora, C. eugenioides and C. racemosa plants were maintained at Centro de Investigação das Ferrugens do Cafeeiro (CIFC) in greenhouse conditions.

\subsection{Preparation of Plant Material}

Fully expanded leaves of the terminal node (young) and from the second pair (mature) were collected, measured and frozen in liquid nitrogen before being freeze-dried (Ilshin, Lab Co. Ltd., Dongducheon, South Korea) to a constant weight. Dried samples were ground by blending to a fine homogenous powder in a Reutsch MM400 Mixer Mill prior to extraction.

\subsection{Coffee Leaf Extraction}

Extraction of phytochemicals was carried out as described: $200 \mathrm{mg}$ of plant material (lyophilized leaves ground with a mixer mill) was sonicated for $30 \mathrm{~min}$. at room temperature $(35 \mathrm{kHz}$, Bandelin sonorex, Berlin, Germany) in $4 \mathrm{~mL}$ of $\mathrm{EtOH} / \mathrm{H}_{2} \mathrm{O}(50: 50, v / v)$, containing sodium metabisulfite $(1 \mathrm{~g} / \mathrm{L})$. After centrifugation $\left(5 \mathrm{~min}, 8602 \times \mathrm{g}\right.$ RCF at $10{ }^{\circ} \mathrm{C}$, Allegra 64R Centrifuge, Beckman Coulter, Indianapolis, IN, USA) the ethanolic extract was collected and filtered (Phenex, NY $0.20 \mu \mathrm{m}$ porosity, Phenomenex, Torrance, CA, USA) and kept at $4{ }^{\circ} \mathrm{C}$ before analysis. Each extraction was performed at least in duplicate. Each sample was characterized by its mean content of alkaloids, chlorogenic acids and xanthones, expressed as $\mathrm{mg} / \mathrm{g}$ of leaves (dry weight).

\subsection{Validation of the Ultra-High Performance Liquid Chromatography-DAD method}

The evaluation of the limit of detection (LOD) and limit of quantitation (LOQ) for the individual compounds were calculated based on a signal-to-noise (S/N) ratio >3 (LOD) and S/N >10 (LOQ), using the following formulas:

$$
\begin{array}{r}
\mathrm{LOD}=3 \times \mathrm{c} /(\mathrm{S} / \mathrm{N}) \\
\mathrm{LOQ}=10 \times \mathrm{c} /(\mathrm{S} / \mathrm{N})
\end{array}
$$

where $\mathrm{c}=$ concentration .

The repeatability of the peak areas was check at all the concentrations for each compound by calculating the relative standard deviation (RSD) and percent bias value for 5 replicates over the course of 3 days as follows: RSD $(\%)=($ standard deviation $/$ mean $) \times 100$ and Bias $(\%)=[($ calculated concentration - theoretical concentration)/theoretical concentration] $\times 100$. 


\subsection{Description of Ultra-High Performance Liquid Chromatography-DAD}

Quantitative analyses were carried out using a 1290 UHPLC system (Agilent Technologies, Santa Clara, CA, USA) equipped with a degasser, quaternary pump, column thermostat and diode array detector (DAD) operating at 254, 258, 273, 305, and 324 nm. A Kinetex ${ }^{\circledR} 2.6 \mu \mathrm{m}$ XB-C18 $100 \AA$, LC column $30 \times 2.1 \mathrm{~mm}$ (Phenomenex, Torrance, CA, USA) was used at room temperature. The elution system $\left(0.4 \mathrm{~mL} \mathrm{~min}^{-1}\right)$ involved two filtered solvents, namely acetonitrile (solvent $\mathrm{A}$ ) and $0.1 \%$ formic acid in water (solvent B) with the following gradient: $0 \mathrm{~min}, 99 \%$ solvent B; 0-6 min, 90\%; 6-10 min, $60 \% ; 10-11 \mathrm{~min}, 99 \%$; isocratic, 99\% until $14 \mathrm{~min}$. The injection volume was $2 \mu \mathrm{L}$. The identification of alkaloids, chlorogenic acids and xanthones were achieved by comparison of specific retention times of standard solutions; quantitative determination was performed using calibration curves of standards. Standards stock solutions were prepared in $\mathrm{MeOH}: \mathrm{H}_{2} \mathrm{O}(3: 7)$ over the concentration range 1 and $450 \mathrm{mg} / \mathrm{L}$. Different diluted solutions were prepared from stock solutions with water. Stock and diluted solutions were kept refrigerated at $4{ }^{\circ} \mathrm{C}$ before use.

\subsection{Description of Ultra-High Performance Liquid Chromatography Electrospray Ionization Mass Spectrometry}

UHPLC-ESI-MS analysis was conducted on an Agilent 1290 HPLC (Santa Clara, CA, USA), coupled to a Sciex Triple Quad 4500 (Farmingham, MA, USA). Chromatographic separation of ethanol extracts was conducted using a column of the same type, and dimensions as for analytical UHPLC, with identical gradient elution. MS Electrospray Ionisation Source was operating in negative mode, acquiring in Multiple Reaction Monitoring (MRM). Operating conditions were optimized using a 5-CQA standard solutions. The source temperature was $350^{\circ} \mathrm{C}$. Specific compounds transitions were confirmed based on literature data and comparison with reference standard solutions. Taking into consideration that, according to the literature [27] cis isomers show the same fragmentation pattern of the corresponding trans isomers, the presence of possible cis isomers was confirmed by comparison of specific fragmentation of the UV treated solutions.

\subsection{Statistical Analysis}

Data were analyzed by one-way analysis of variance (ANOVA) using XLStat 2018 (Addinsoft, Bordeaux, France). Significant differences among samples were compared using Tukey's tests. Data were expressed as means $\pm \mathrm{SD}$ and $p<0.05$ represents statistically significant difference.

\section{Results and Discussion}

\subsection{Validation of the UHPLC-DAD Method}

In this work, ultra-high pressure liquid chromatography (UHPLC) was preferred over high pressure liquid chromatography (HPLC) as the analytical tool, because it is more economical and offers higher sensitivity. UHPLC uses shorter and narrower columns filled with small-sized particles $(<3 \mu \mathrm{m})$ in combination with very high pressure, resulting in lower volumes of solvents used, shorter analysis time, increased sensitivity, and excellent resolution [28]. The use of the diode array detector (DAD) coupled to the UHPLC ensures the clear identification of each component. HPLC coupled with a DAD is the most reported method for the quantification of the main secondary metabolites present in coffee leaves [11-13], in which each run averaging over $25 \mathrm{~min}$. The use of UHPLC-DAD in this work has allowed to complete the analysis of each run in $14 \mathrm{~min}$, considerably improving the length of time require for this analysis, and consequently decreasing the limit of detection. This is the first time that the use of UHPLC is being reported for the evaluation of coffee leaves.

All the main compounds under investigation, trigonelline, theobromine, caffeine, 3-CQA, 5-CQA, 4-CQA, 3,4-DiCQA, 3,5-DiCQA, 4,5-DiCQA, mangiferin, and isomangiferin, were successfully separated and identified by UHPLC-DAD and their structure confirmed via MS/MS analyses of specific transitions (Table S2). The quantification of alkaloids, CGAs and xanthones in the dry coffee leaf samples was done by UV-Vis spectroscopy, using the DAD detector, by monitoring the absorbance at 
the maximum wavelength for each compounds, i.e., $254 \mathrm{~nm}$ for trigonelline, $258 \mathrm{~nm}$ for mangiferin and isomangiferin, $273 \mathrm{~nm}$ for theobromine and caffeine and $324 \mathrm{~nm}$ for the CGA. Calibration curves were prepared in the range of concentration comprised between 4 and $200 \mu \mathrm{g} / \mathrm{mL}$ and the data were fitted using least-square linear regression. Good linearity $\left(r^{2}>0.9987\right)$, expressed as the determination coefficient, was obtained for all compounds, as shown in Table 1.

Table 1. Ultra-high-performance liquid chromatography (UHPLC)-UV method optimization results.

\begin{tabular}{|c|c|c|c|c|c|c|c|}
\hline \multirow{2}{*}{ Analyte } & \multirow{2}{*}{$\begin{array}{l}\lambda_{\max } \\
(\mathrm{nm})\end{array}$} & \multirow{2}{*}{$\begin{array}{l}\text { Retention Time } \\
\text { (min) }\end{array}$} & \multicolumn{3}{|c|}{ Calibration Curve } & \multirow{2}{*}{$\begin{array}{l}\operatorname{LOD}^{1} \\
(\mu \mathrm{g} / \mathrm{mL})\end{array}$} & \multirow{2}{*}{$\begin{array}{c}\mathrm{LOQ}^{2} \\
(\mu \mathrm{g} / \mathrm{mL})\end{array}$} \\
\hline & & & Intercept & Slope & $r^{2}$ & & \\
\hline Trigonelline & 254 & 0.60 & 9.025 & 5.375 & 0.9996 & 0.110 & 0.365 \\
\hline $\begin{array}{l}\text { Mangiferin } \\
\text { Isomangiferin }\end{array}$ & 258 & $\begin{array}{l}7.71 \\
7.85\end{array}$ & 4.986 & 16.409 & 0.9987 & 0.025 & 0.083 \\
\hline $\begin{array}{l}\text { Theobromine } \\
\text { Caffeine }\end{array}$ & 273 & $\begin{array}{l}3.65 \\
6.24 \\
\end{array}$ & $\begin{array}{c}1.482 \\
32.541\end{array}$ & $\begin{array}{l}17.420 \\
14.371\end{array}$ & $\begin{array}{l}0.9999 \\
0.9999\end{array}$ & $\begin{array}{l}0.023 \\
0.003\end{array}$ & $\begin{array}{l}0.075 \\
0.012\end{array}$ \\
\hline $\begin{array}{c}\text { 3-CQA } \\
\text { 5-CQA } \\
\text { 4-CQA } \\
\text { 3,4-DiCQA } \\
\text { 3,5-DiCQA } \\
\text { 4,5-DiCQA }\end{array}$ & 324 & $\begin{array}{c}4.49 \\
6.18 \\
6.66 \\
9.88 \\
10.05 \\
10.41\end{array}$ & $\begin{array}{c}8.217 \\
23.912 \\
0.710 \\
1.354 \\
0.771 \\
-1.908\end{array}$ & $\begin{array}{c}8.924 \\
8.487 \\
9.330 \\
8.913 \\
11.102 \\
11.486\end{array}$ & $\begin{array}{l}0.9999 \\
0.9998 \\
0.9999 \\
0.9995 \\
0.9999 \\
0.9993\end{array}$ & $\begin{array}{l}0.066 \\
0.089 \\
0.044 \\
0.016 \\
0.108 \\
0.057\end{array}$ & $\begin{array}{l}0.220 \\
0.297 \\
0.145 \\
0.054 \\
0.359 \\
0.190\end{array}$ \\
\hline
\end{tabular}

${ }^{1} \mathrm{LOD}=$ Limit of detection, ${ }^{2} \mathrm{LOQ}=$ Limit of quantification.

The limit of detection (LOD) and the limit of quantification (LOQ), the minimum amount of an analyte in a sample that can be detected and quantified are important parameters for the comparison of different sensitivities of analytical methods. However, literature data on the quantification of main secondary metabolites present in coffee leaves do not often provide this information. To the best of our knowledge only one study has reported values of LOD and LOQ when quantifying mangiferin in coffee leaves [12].

The quantification method used in this work provided values of LODs for all analytes, and these were found to be ranging from 0.003 to $0.110 \mu \mathrm{g} / \mathrm{mL}$, which are considerably lower than the concentrations reported until now in literature, for coffee leaf samples [10-12]. Comparison of the LOD data can only be done with metabolites isolated from different matrices, other than coffee leaves. Trigonelline showed comparable LOD $(0.11 \mathrm{mg} / \mathrm{L})$ to a HPLC-MS method used to analyze roasted and green coffee beans [29]. For caffeine and theobromine, the LODs were found to be the lowest, in the range of $0.003-0.023 \mu \mathrm{g} / \mathrm{mL}$, which are consistent with the one reported for caffeine in coffee beans determined by HPLC-MS [29]. The LODs for CGAs were in the range of 0.044-0.108 $\mu \mathrm{g} / \mathrm{mL}$, similar to the values obtained when brewed coffee was analyzed using a HPLC-DAD method [30]. The LODs of mangiferin and isomangiferin $(0.025 \mathrm{mg} / \mathrm{L})$ were found to be at least 50 times lower than the values reported for HPLC-UV method used to analyse coffee leaves [12], proving the higher sensitivity of UHPLC over HPLC.

The intra-assay (intra-day) and inter-assay (inter-day) variability of the method are parameters that evaluate the accuracy and precision of an analytical method, which is characterized by the closeness between the measured values obtained by replicate measurements. In this study, the intraand inter-assay variability were assessed by analyzing the aqueous standards of the analytes of interest. The precision was evaluated through intra-day and inter-day relative standard deviation percentage (RSD) and percent bias values for three different concentration levels, as shown in Table S3. The results showed an RSD lower than $2.98 \%$ for the intra- and inter-assay for all the analytes, consistent with literature data $[12,29,30]$. The percent bias value lower than 15\% (absolute value) was verified for all the analytes, with the exception of 4,5-DiCQA at the lowest concentration $(-19.89 \%$ and $-21.39 \%$, intra and inter-assay respectively). These data confirm the precision and accuracy of the developed 
analytical method, which follows the required acceptance criteria of $\operatorname{RSD}<15 \%$, and the percent bias value within $\pm 15 \%$.

\subsection{Quantification of Alkaloids, Chlorogenic acids, Mangiferin, and Isomangiferin in Coffee Leaves}

The UHPLC-DAD quantitative method described here allowed the separation of chlorogenic acids (CQAs, diCQAs, p-CoQAs, FQAs, and the correspondent cis isomers of CQAs, $p$-CoQAs, and diCQAs), alkaloids (caffeine, theobromine, and trigonelline), mangiferin and isomangiferin (Figure 1). Trigonelline, theobromine, caffeine, mono- and di-chlorogenic acids, mangiferin, and isomangiferin were quantified using the calibration curves previously described (Table 1). FQAs, $p$-CoQAs and cis isomers were identified by UHPLC-MS/MS analysis. Analysis by ESI-MS/MS was performed only for qualitative purposes, due to lack of available commercial or synthesized standards of certified purity (Table S2). The MRM transitions used to identify the different minor CGAs have been previously reported [31].

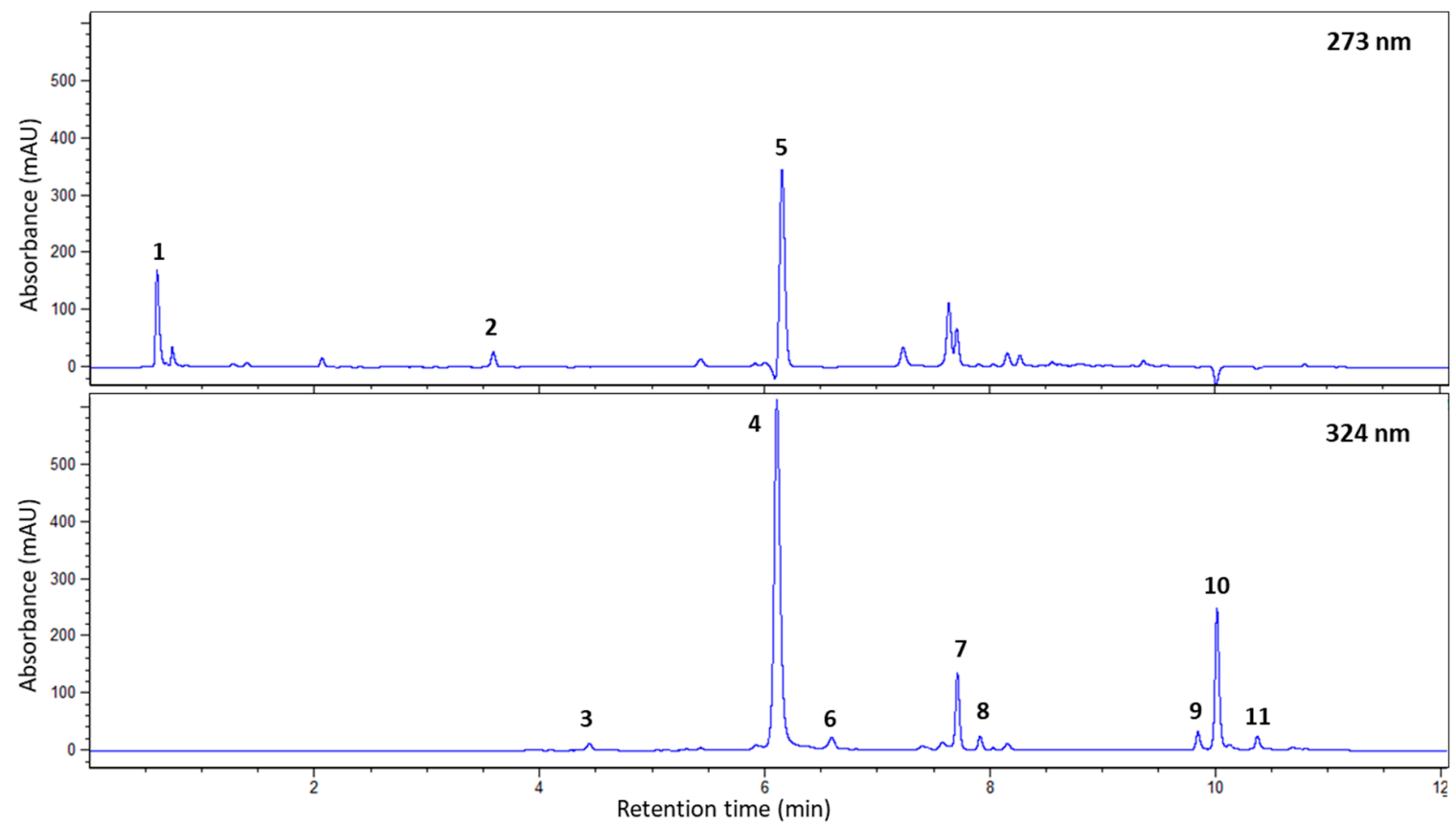

Figure 1. This UHPLC profile of $a$ C. arabica young leaf extract. Absorption profile obtained at 273 and $324 \mathrm{~nm}$ using the technical conditions for quantitative analysis. Peak $1=$ trigonelline; Peak 2 = theobromine; Peak 3 = 3-caffeoylquinic acid (3-CQA); Peak 4 = 5-caffeoylquinic acid (5-CQA); Peak 5 = caffeine; Peak 6 = 4-caffeoylquinic acid (4-CQA); Peak 7 = mangiferin; Peak 8 = isomangiferin; Peak 9 = 3,4-dicaffeoylquinic acid (3,4 diCQA); Peak 10 = 3,5-dicaffeoylquinic acid (3,5-diCQA); Peak $11=4,5$-dicaffeoylquinic acid (4,5-diCQA).

\subsubsection{Alkaloids}

Trigonelline, a pyridine alkaloid, was found in all the coffee leaf species analysed (Table 2). Its content in C. arabica young leaves $(11.718 \mathrm{mg} / \mathrm{g}$ of dry matter) is in accordance with previous reported data (10.990 mg/g of dry matter) [32]. Young leaves of both genotypes of C. racemosa and C. eugenioides showed also a trigonelline content (11.188 and $11.090 \mathrm{mg} / \mathrm{g}$ and, 14.596 and $9.932 \mathrm{mg} / \mathrm{g}$ of dry matter, respectively) similar to that of $C$. arabica young leaves, while $C$. canephora young leaves showed a lower content in trigonelline ( 7.879 and $4.817 \mathrm{mg} / \mathrm{g}$ of dry matter). The mature leaves showed a decrease of trigonelline content in comparison to young leaves, ranging from $12.1 \%$ to $24.1 \%$, in C. canephora, C. eugenioides, and C. racemosa, which is in agreement with 6-month-old C. arabica seedlings previously reported (up to $23.6 \%$ ) [32]. 
The content of trigonelline in young leaves, ranging from $0.48 \%$ to $1.46 \%$ on dry matter basis $(\mathrm{dmb})$ is similar to the content found in the green coffee beans of the species studied [33]. This evidence suggests that a coffee leaf tea would contain at least double the amount of trigonelline, considered a novel phytoestrogen in coffee beans [22], compared to the average quantity present in a coffee beverage; this can be easily justified as up to $70 \%$ of its content is lost during the roasting process to form compounds that are responsible for both flavour and bioactivity of coffee [34].

Caffeine and theobromine were the only purine alkaloids only found in three species of coffee leaves, namely C. arabica, C. canephora, and C. racemosa. The absence of the theophylline in coffee leaves is in accordance with previous studies [10,35], as the catabolism of caffeine is very low, leading to loss of caffeine in coffee leaves. Caffeine was only detected in the leaves of C. arabica and C. canephora, with concentrations of $14.936 \mathrm{mg} / \mathrm{g}$ and $10.043-13.186 \mathrm{mg} / \mathrm{g}$ (young leaves) and $5.993 \mathrm{mg} / \mathrm{g}$ and $2.805-7.527 \mathrm{mg} / \mathrm{g}$ (mature leaves) of dry matter respectively (Table 2). The concentrations of caffeine in young leaves are consistent with previous findings [36] and comparable to the amount present in C. arabica green coffee beans [33]. Although the similar concentration of caffeine found in both C. arabica and C. canephora leaves, these are known to differ considerably between green/roasted C. arabica and C. canephora beans, a higher variability in the caffeine content of C. canephora than in C. arabica has been often reported as a result of different genotypes and/or climatic conditions $[33,37]$. When mature leaves were analyzed, a decrease in caffeine was observed, ranging between $43 \%$ and $72 \%$, which was proportional to the decrease in caffeine content of $C$. arabica leaves observed by Ashihara et al. [10]. Although more recently a slow rate of caffeine catabolism was observed, the fact that caffeine biosynthesis occurs mainly in young leaves may explain the variation observed in mature leaves [38]. The presence of very small amounts of caffeine in our samples of C. eugenioides and C. racemose leaves (inferior to the LOD, determined for the UHPLC-DAD method) could be the consequence of a rapid catabolism of caffeine accompanied by a slow rate of caffeine biosynthesis in these species.

Theobromine, was also found only in C. arabica and C. canephora and in one genotype of C. racemosa (Table 2). Young leaves showed a theobromine content of $0.811 \mathrm{mg} / \mathrm{g}$ of dry matter in C. arabica, half of the content reported in a previous study in fresh matter [10]. In C. canephora young leaves, a high variability found on the theobromine content $(0.169-4.142 \mathrm{mg} / \mathrm{g}$ of dry matter) between the two genotypes studied has been also reported [35]. In mature leaves, a decrease of $94 \%$ of the theobromine content was verified for C. arabica, which has been previously observed by Ashihara et al. [10], and a decrease ranging from $73 \%$ to $81 \%$ of the theobromine in C. canephora which is comparable to the decrease verified in C. arabica leaves. In the low caffeine content species, C. racemosa and C. eugenioides, theobromine was surprisingly found in moderate amounts $(1.53 \mathrm{mg} / \mathrm{g}$ of dry matter) in the leaves of one genotype of C. racemosa, once has been reported the absence of this metabolite in C. racemosa leaves [39]. A decrease of theobromine content with leaf aging was also observed in C. racemose leaves.

As a result of this thorough analysis, it can be concluded that teas produced from the coffee leaves of C. eugenioides and C. racemosa can be considered naturally decaffeinated products. The low content of caffeine detected $(<0.1 \%$ on a dry basis), in accordance with the current accepted coffee regulations [40], allows such labelling without the need for a decaffeination process. This is significant, as it would impact directly the economics of coffee production, reducing considerably the price of this type of product for people who are sensitive to caffeine or wish to limit its intake. 
Table 2. Alkaloids composition of coffee leaves (mg/g of dry matter \pm SD).

\begin{tabular}{|c|c|c|c|c|c|c|c|c|c|}
\hline \multirow{2}{*}{ Species } & \multirow{2}{*}{$\begin{array}{l}\text { Coffee } \\
\text { Genotype }\end{array}$} & \multicolumn{2}{|c|}{ Trigonelline } & \multicolumn{2}{|c|}{ Theobromine } & \multicolumn{2}{|c|}{ Caffeine * } & \multicolumn{2}{|c|}{ Total Alkaloids } \\
\hline & & Young & Mature & Young & Mature & Young & Mature & Young & Mature \\
\hline C. arabica & var. Bourbon & $11.718 \pm 0.544$ & $2.955 \pm 0.207$ & $0.811 \pm 0.029$ & $0.045 \pm 0.005$ & $14.936 \pm 0.019 \mathbf{a}$ & $5.993 \pm 0.508 \mathbf{e}$ & $27.465 \pm 0.592$ & $8.993 \pm 0.720$ \\
\hline \multirow{2}{*}{ C. canephora } & CIFC 2975 & $7.879 \pm 0.209$ & $5.979 \pm 0.017$ & $0.169 \pm 0.001$ & $0.046 \pm 0.000$ & $13.186 \pm 0.423 \mathbf{b}$ & $7.527 \pm 0.032 \mathrm{~d}$ & $21.234 \pm 0.633$ & $13.552 \pm 0.049$ \\
\hline & CIFC 829/1 & $4.817 \pm 0.019$ & $4.157 \pm 0.300$ & $4.142 \pm 0.040$ & $0.806 \pm 0.076$ & $10.043 \pm 0.043 c$ & $2.805 \pm 0.077 \mathbf{f}$ & $19.002 \pm 0.102$ & $7.769 \pm 0.454$ \\
\hline \multirow{2}{*}{ C. eugenioides } & CIFC 1634/11 & $14.596 \pm 0.143$ & $11.232 \pm 0.001$ & - & - & - & - & $14.596 \pm 0.143$ & $11.232 \pm 0.001$ \\
\hline & CIFC 241/43 & $9.932 \pm 0.021$ & $7.781 \pm 0.002$ & - & - & - & - & $9.932 \pm 0.021$ & $7.781 \pm 0.002$ \\
\hline \multirow{2}{*}{ C. racemosa } & CIFC 1693/76 & $11.188 \pm 0.222$ & $9.836 \pm 0.177$ & $1.532 \pm 0.026$ & $0.549 \pm 0.003$ & - & - & $12.721 \pm 0.245$ & $10.385 \pm 0.180$ \\
\hline & CIFC 13969 & $11.090 \pm 0.245$ & $9.736 \pm 0.092$ & - & - & - & - & $11.090 \pm 0.245$ & $9.736 \pm 0.092$ \\
\hline
\end{tabular}

Data are shown as means \pm SD. Total alkaloids are the sum of trigonelline, theobromine and caffeine. ${ }^{*}$ Total caffeine concentrations are labelled with different letters (a-f) to highlight

values that are considered statistically different $(p<0.5)$. 


\subsubsection{Chlorogenic Acids}

As shown in Table 3, the total CGAs content in young leaves, calculated as the sum of mono- and di-caffeoylquinic acids, varied from 35.732 to $80.836 \mathrm{mg} / \mathrm{g}$ of dry matter; C. arabica and C. eugenioides showed the highest content, while C. canephora and C. racemosa showed the lowest content in CGAs, consistent with the trend recently reported by Campa et al. [11]. The overall high content of CGAs in young coffee leaves is in agreement with previous studies on different coffee leaf species $[11,13,16,17]$. Specifically, a high content of 5-CQA followed by 3,5-DiCQA (2 to 8 times lower than 5-CQA) was also found, the sum of which accounts for $82 \%$ of the total amount of CGAs present in coffee leaves; this trend had already been reported $[13,16,17]$. However, in young $C$. racemosa leaves a lower content 3,5-DiCQA was observed, while 3-CQA and 4-CQA contents were considerably higher than the values obtained for the young leaves of the other coffee species analysed in this study.

The mature leaves showed a decrease in concentration of CGAs that ranged from $40.8 \%$ to $51.7 \%$ less than the concentration in their respective young leaves, with the exception of C. racemosa CIFC 1693/76 which showed a lower decrease in the concentration of CGAs of (27.4\%) and C. arabica Bourbon which showed a higher decrease in the concentration of CGAs (78\%) with aging (Table 3). More specifically, the decrease in 5-CQA content observed ranged from $26.3 \%$ to $48.5 \%$, with the exception for C. arabica leaves (74.2\%), while the decrease in 3,5-DiCQA content observed was almost the double $(46.3 \%-97.2 \%)$. The decrease in content of the other CGAs was also observed but to lesser extent, $12.5 \%$ to $31.9 \%$ less than in young leaves. These data show that 3,5-DiCQA is the compound whose concentration is most susceptible to aging, followed by 5-CQA, which was also previously verified [16,17], and the other chlorogenic acids present in a less extent, suggesting that CQAs and DiCQAs might undergo different metabolic processes of degradation during leaf aging. The same pattern in change of the concentration with of the different CGAs was also verified in the whole fruit and pericarp of C. arabica and C. canephora [41]. Interestingly, in C. arabica var. Bourbon, the content of 3-CQA and 4-CQA increased with aging, $62 \%$ and $17 \%$ respectively, as recently reported by Campa et al. [17].

The quantitative comparison of the content of CGAs of coffee leaf samples from different studies is often challenging, as the biosynthesis of these secondary metabolites is not only dependent on the age of the plant from which the leaves were collected but also on the environmental/greenhouse conditions. A recent study demonstrated a significant variation of the concentration of the different CGAs in C. arabica leaves originating from different regions in Brazil [13]. This highlights the importance of citing the environmental context in which samples are grown and their development stage when reporting quantitative analysis of coffee leaves. Nevertheless, a general trend can be observed within literature data, in which 5-CQA results as the most abundant chlorogenic acid, followed by 3,5-DiCQA and the rest of CGAs, namely 3-CQA, 4-CQA, 3,4-DiCQA, and 4,5-DiCQA [13,16].

A number of other chlorogenic acids, present in very low concentration, were also identified by ESI-MS/MS (Table S2) due to the absence of HPLC standards; these were 3-p-coumaroylquinic acid (3- $p$ CoQA), 4- $p$-coumaroylquinic acid (4- $p$ CoQA), 5- $p$-coumaroylquinic acid (5- $p$ CoQA), 4-feruoylquinic acid (4-FQA), 5-feruoylquinic acid (5-FQA), cis 3-caffeoylquinic acid (cis 3-CQA), cis 3-p-coumaroylquinic acid (cis 3-pCoQA), cis 4-caffeoylquinic acid (cis 4-CQA), cis 4-p-coumaroylquinic acid (cis 4-pCoQA), cis 5-caffeoylquinic acid (cis 5-CQA), cis 5-p-coumaroylquinic acid (cis 5-pCoQA), and one of the cis isomers of 3,5-DiCQA. The presence of these minor CGAs and the absence of 3-feruoylquinic acid (3-FQA) has been previously observed [11]. Particularly, the presence of cis the isomers of CGAs, which are present in very low concentrations in coffee beans, is expected as coffee leaves are naturally more exposed to ultraviolet (UV) light compared to coffee beans. However, the concentration of cis isomers of CGA present in all the analysed samples was too low to allow their quantification, possibly due to the limited exposure of coffee leaves to UV light, as a result of being grown inside greenhouses [11]. 
Table 3. Chlorogenic acids (CGAs) composition of coffee leaves (mg/g of dry matter \pm SD).

\begin{tabular}{|c|c|c|c|c|c|c|c|c|c|}
\hline \multirow{2}{*}{ Species } & \multirow{2}{*}{$\begin{array}{l}\text { Coffee } \\
\text { Genotypes }\end{array}$} & \multicolumn{2}{|c|}{ 3-CQA } & \multicolumn{2}{|c|}{ 5-CQA } & \multicolumn{2}{|c|}{ 4-CQA } & \multicolumn{2}{|c|}{ 3,4-DiCQA } \\
\hline & & Young & Mature & Young & Mature & Young & Mature & Young & Mature \\
\hline C. arabica & var. Bourbon & $0.881 \pm 0.070$ & $1.429 \pm 0.103$ & $56.790 \pm 3.640$ & $14.678 \pm 1.617$ & $1.586 \pm 0.110$ & $1.853 \pm 0.155$ & $1.798 \pm 0.133$ & $0.431 \pm 0.040$ \\
\hline \multirow{2}{*}{ C. canephora } & CIFC 2975 & $0.906 \pm 0.031$ & $0.873 \pm 0.002$ & $22.880 \pm 0.796$ & $13.874 \pm 0.033$ & $0.906 \pm 0.006$ & $1.035 \pm 0.008$ & $1.003 \pm 0.007$ & $0.441 \pm 0.000$ \\
\hline & CIFC 829/1 & $1.395 \pm 0.002$ & $1.217 \pm 0.031$ & $29.458 \pm 0.155$ & $16.346 \pm 0.798$ & $3.850 \pm 0.003$ & $2.602 \pm 0.175$ & $1.001 \pm 0.007$ & $0.498 \pm 0.018$ \\
\hline \multirow{2}{*}{ C. eugenioides } & CIFC 1634/11 & $0.521 \pm 0.003$ & $0.402 \pm 0.004$ & $49.581 \pm 0.299$ & $31.451 \pm 0.538$ & $3.148 \pm 0.000$ & $2.872 \pm 0.040$ & $0.549 \pm 0.001$ & $0.257 \pm 0.005$ \\
\hline & CIFC 241/43 & $0.716 \pm 0.006$ & $0.450 \pm 0.005$ & $34.283 \pm 0.657$ & $17.667 \pm 0.200$ & $5.096 \pm 0.039$ & $3.388 \pm 0.040$ & $0.421 \pm 0.012$ & $0.044 \pm 0.000$ \\
\hline \multirow[b]{2}{*}{ C. racemosa } & CIFC 1693/76 & $3.007 \pm 0.030$ & $2.816 \pm 0.018$ & $21.123 \pm 0.129$ & $15.574 \pm 0.002$ & $8.359 \pm 0.112$ & $6.546 \pm 0.099$ & $0.797 \pm 0.009$ & $0.628 \pm 0.032$ \\
\hline & CIFC 13969 & $1.618 \pm 0.016$ & $2.234 \pm 0.006$ & $46.145 \pm 0.752$ & $24.781 \pm 0.129$ & $8.232 \pm 0.050$ & $6.282 \pm 0.032$ & $2.044 \pm 0.101$ & $1.768 \pm 0.043$ \\
\hline \multirow{2}{*}{ Species } & \multirow{2}{*}{$\begin{array}{l}\text { Coffee } \\
\text { Genotypes }\end{array}$} & \multicolumn{2}{|c|}{ 3,5-DiCQA } & \multicolumn{2}{|c|}{ 4,5-DiCQA } & \multicolumn{2}{|c|}{ Total CGAs * } & & \\
\hline & & Young & Mature & Young & Mature & Young & Mature & & \\
\hline C. arabica & var. Bourbon & $11.266 \pm 0.591$ & $0.318 \pm 0.030$ & $1.217 \pm 0.045$ & $0.499 \pm 0.067$ & $73.539 \pm 4.590 \mathbf{b}$ & $19.208 \pm 2.012 \mathbf{i}$ & & \\
\hline \multirow{2}{*}{ C. canephora } & CIFC 1693/76 & $9.952 \pm 0.184$ & $2.994 \pm 0.034$ & $0.085 \pm 0.001$ & $0.095 \pm 0.003$ & $35.732 \pm 1.027 \mathrm{~g}$ & $19.312 \pm 0.080 \mathbf{i}$ & & \\
\hline & CIFC 13969 & $11.035 \pm 0.070$ & $1.934 \pm 0.019$ & $0.185 \pm 0.003$ & $0.066 \pm 0.013$ & $46.924 \pm 0.239 \mathrm{~d}$ & $22.663 \pm 1.042 \mathbf{i}$ & & \\
\hline \multirow{2}{*}{ C. eugenioides } & CIFC 2975 & $6.513 \pm 0.136$ & $3.495 \pm 0.302$ & $0.704 \pm 0.118$ & $0.344 \pm 0.019$ & $40.503 \pm 0.533$ ef & $29.402 \pm 0.473 \mathbf{h}$ & & \\
\hline & CIFC 829/1 & $20.309 \pm 0.423$ & $6.739 \pm 0.001$ & $2.488 \pm 0.080$ & $2.308 \pm 0.105$ & $80.836 \pm 1.422 \mathbf{a}$ & $44.112 \pm 0.180 \mathrm{de}$ & & \\
\hline \multirow{2}{*}{ C. racemosa } & CIFC 1634/11 & $6.837 \pm 0.065$ & $1.348 \pm 0.011$ & $1.150 \pm 0.013$ & $0.272 \pm 0.003$ & $61.782 \pm 0.382 \mathrm{c}$ & $36.603 \pm 0.600 \mathrm{fg}$ & & \\
\hline & CIFC $241 / 43$ & $2.388 \pm 0.068$ & $0.084 \pm 0.000$ & $0.914 \pm 0.033$ & $0.091 \pm 0.003$ & $43.818 \pm 0.814 \mathbf{d e}$ & $21.724 \pm 0.248 \mathbf{i}$ & & \\
\hline
\end{tabular}

Data are shown as means \pm SD. Total CGAs are the sum of 3-, 4-, 5-CQA and 3,4-; 3,5-; 4,5-diCQA. * Total CGAs concentrations are labelled with letters (a-i) that group values that are

considered not statistically different $(p>0.5)$ 
The total amount of CGAs accumulated in the young leaves (dry matter) was found to be $7.4 \%$ in C. arabica, 3.6\% and $4.7 \%$ in C. canephora, $4.4 \%$ and $6.2 \%$ in C. eugenioides, and $4.1 \%$ and $8.1 \%$ in C. racemosa. It is noteworthy that C. canephora, C. eugenioides, and C. racemosa, showed quantities of CGAs accumulated in their young leaves similar to the amounts present in green coffee beans with the exception of $C$. canephora young leaves, which showed half of the quantity of CGAs present in its coffee beans, as previously observed for caffeine [42].

The results suggest that coffee leaf tea could have health benefits comparable to green coffee beans due to the similar concentrations of CGAs, which are twofold higher than in roasted coffee. During roasting a significant portion of these compounds are transformed into derivatives that are responsible for coffee flavour [34]. C. eugenioides or C. racemosa have very low bean yield and therefore are less economically relevant species in coffee production; for this reason, they could be used instead for coffee leaf tea preparation, without compromising the coffee bean process of more economically relevant species, such as C. arabica and C. canephora.

In traditional Chinese medicine the flower and buds of Lonicera japonica (Japanese honeysuckle) and, the leaves of Eucommia ulmoides are widely used for their beneficial biological properties, due to a high concentration of CGAs present in these plant materials [43]. In fact, chlorogenic acid has been used in Chinese Pharmacopeia as a biomarker to characterise the quality of Lonicera flower in terms of biological activities, making it the main active compound responsible for the beneficial biological properties [44]. The concentration of CGAs found in L. japonica ranges from 20 to $60 \mathrm{mg} / \mathrm{g}$ of dry matter depending on the habitat [43], which is comparable to the concentration of CGAs found in all the coffee leaf species analysed here. Therefore, from an application point of view, coffee leaf tea should provide similar health benefits.

\subsubsection{Xanthones}

Both mangiferin and isomangiferin were only found in C. eugenioides and C. arabica leaves. Young leaves showed a mangiferin content of $14.714 \mathrm{mg} / \mathrm{g}$ of dry matter in C. arabica leaves and, 53.875 and $76.686 \mathrm{mg} / \mathrm{g}$ of dry matter in C. eugenioides (Table 4), which are comparable to the levels of mangiferin detected in the leaves of mango trees [20], one of the main sources of mangiferin. As in the case of CGAs content, also here a quantitative comparison of the content of xanthones among different studies is challenging, as a high variability has been observed among different samples of C. arabica leaves from different regions in Brazil and Costa Rica, suggesting that biosynthesis of these secondary metabolites is largely dependent on environmental conditions [12,13]. For instance, the mangiferin content presently reported for young $C$. arabica leaves was found to be at least two times higher than the mangiferin content reported by Campa et al. [11] and de Almeida et al. [13], and from two times higher to three times lower than the mangiferin content reported by Trevisan et al. [12]. However, the concentration of mangiferin found on C. eugenioides leaves by Campa et al. [11] is comparable to the concentration of mangiferin found in this study.

Table 4. Xanthones composition of coffee leaves (mg/g of dry matter $\pm \mathrm{SD}$ ).

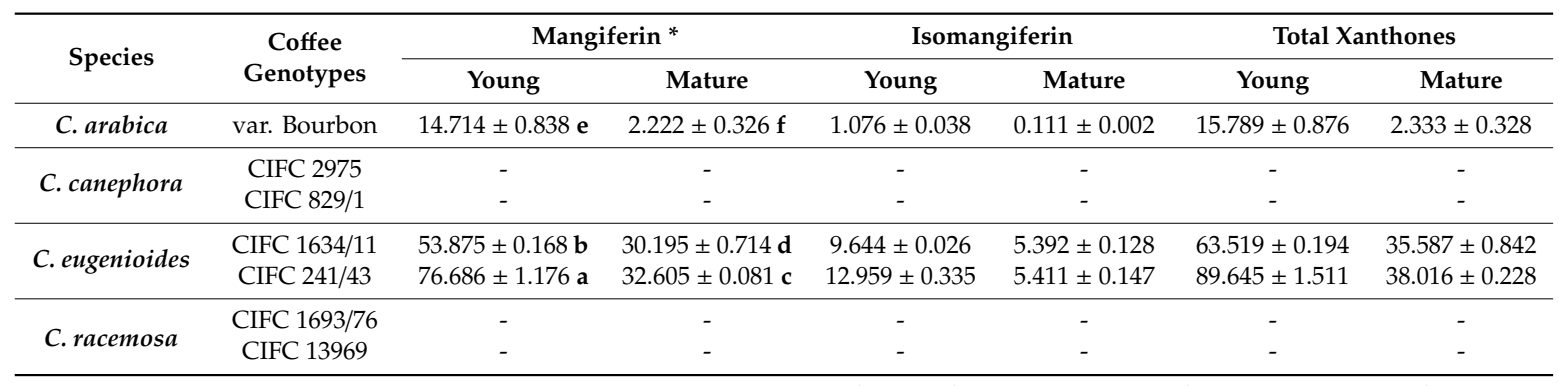

Data are shown as means \pm SD. Total xanthones are the sum of mangiferin and isomangiferin. ${ }^{*}$ Total mangiferin concentrations are labelled with different letters $(a-f)$ to highlight values that are considered statistically different $(p<0.5)$. 
The identification of isomangiferin in the ethanol extracts, which display the typical UV spectrum of mangiferin, was determined from the mass spectrum which showed the same fragmentation pattern of its corresponding isomer, mangiferin. The quantification of isomangiferin was also performed using the calibration curve of mangiferin from the UHPLC-UV chromatograms at $258 \mathrm{~nm}$. Young leaves showed an isomangiferin content of $1.076 \mathrm{mg} / \mathrm{g}$ of dry matter in C. arabica leaves, $20 \%$ more than the content verified by Trevisan et al. [12], and $9.64 \mathrm{mg} / \mathrm{g}$ and $12.96 \mathrm{mg} / \mathrm{g}$ of dry matter in C. eugenioides (Table 4). Additionally, the concentration of isomangiferin was six times lower than mangiferin, which was also verified by Trevisan et al. [12].

The content of mangiferin and isomangiferin was also analysed during leaf aging, which so far has been only studied in C. arabica leaves by Trevisan et al. [12]. The aging process led to a decrease in the mangiferin and isomangiferin content of about $85 \%$ and $90 \%$, respectively, in C. arabica leaves, which was higher than the decrease previously reported [12]. The $C$. eugenioides mature leaves showed a decrease in the concentration for both xanthones of $44 \%$ in CIFC 1634/11 variety, and 58\% for CIFC $241 / 43$ variety, in comparison to their concentration in young leaves (Table 5). Interestingly, the decrease of both xanthones showed to be comparable to catabolism rates of 5-CQA and 3,5-DiCQA in both coffee species. This observation suggests that most probably, due to the presence of the catechol moiety, mangiferin is catabolized by the same enzymes responsible for the degradation of CGAs in coffee.

Table 5. Decrease of the concentration of 5-CQA, 3,5-DiCQA, and xanthones during leaf aging.

\begin{tabular}{ccccc}
\hline \multirow{2}{*}{ Species } & \multirow{2}{*}{ Coffee Genotypes } & \multicolumn{3}{c}{ Decrease during Leaf Aging (\%) } \\
\cline { 3 - 5 } & & 5-CQA & 3,5-DiCQA & Xanthones \\
\hline C. arabica & var. Bourbon & 74 & 97 & 85 \\
\hline \multirow{2}{*}{ C. eugenioides } & CIFC 241/43 & 37 & 46 & 44 \\
& CIFC 1634/11 & 48 & 67 & 58 \\
\hline
\end{tabular}

These xanthones, which possess high potential as cancer chemopreventive, antioxidant and anti-inflammatory agents [45], are found in high concentrations in M. indica (mango) leaves and Cyclopia genistoides (honeybush) shoots, the latter being widely used in South Africa to prepare infusions and with a growing worldwide market [19]. Honeybush tea is known for its beneficial biological properties demonstrated both in vivo and in vitro models, as a result of the presence of high concentrations of mangiferin [19]. The concentration of mangiferin found in C. genistoides shoots, up to $72.1 \mathrm{mg} / \mathrm{g}$ of dry matter, was found to be comparable to the young leaves of C. arabica and C. eugenioides [19], suggesting that a coffee leaf tea would contain an amount of mangiferin comparable to the one present in honeybush tea, in addition to the high concentration of CGAs in coffee leaves.

The data confirm how an infusion made from young coffee leaves would provide a beverage rich in polyphenols, with potential benefits to human health. Recently, the effect of different tea processing methods on young and mature coffee leaves has been studied and their bioactivities evaluated [25,26]. On one hand, the high content in polyphenols (mainly 5-CQA, 3,5-DiCQA and mangiferin), present in young leaves (C. arabica), is responsible for its high antioxidant and anti-inflammatory activities. On the other hand, the mature leaves (C. arabica) do not shown such a high antioxidant and anti-inflammatory activities, due to the lack of polyphenols, but can potentially diminish high blood pressure and protect against microbial invasion $[25,26]$.

\section{Conclusions}

The present study reports the content of a number of bioactive compounds (trigonelline, xanthines, CGAs, and xanthones) abundant in four different coffee leaf species at different development stages: C. arabica, C. canephora, C. eugenioides, and C. racemosa. Trigonelline, recently considered a novel phytoestrogen in coffee beans, was found in the leaves of all four different coffee species analyzed. In particular, young leaves contain a concentration of trigonelline comparable to the one found in 
green coffee beans, which means that a coffee leaf tea would contain at least double the amount of trigonelline present in coffee, due to its partial degradation during roasting. Caffeine was found in the young leaves of $C$. arabica and C. canephora in similar concentrations to the ones found in the green coffee beans of $C$. arabica, while the quantification in C. eugenioides and C. racemosa proved to be very challenging. A coffee leaf tea produced from C. eugenioides and C. racemosa provides a naturally decaffeinated product potentially useful for consumers' sensitive to caffeine. The last alkaloid found in abundance in coffee leaves was theobromine, which was present in C. arabica and C. canephora with a high variability $(0.17-4.14 \mathrm{mg} / \mathrm{g}$ of dry matter). Surprisingly, theobromine was found in a moderate amount in the leaves of one of the two C. racemosa genotypes (CIFC 1693/76).

All the young leaves of coffee species analyzed showed the presence of CGAs in similar quantities to those that are found in the green coffee beans of their respective species, with the exception of C. canephora that showed half of the CGAs present in green coffee beans. The most abundant CGAs in the majority of the coffee leaf species were 5-CQA and 3,5-DiCQA, which were also the most affected during leaf aging through possibly different metabolic processes of degradation. From an application point of view, a coffee leaf tea shares the same beneficial biological properties present in green coffee beans, due to the high content of CGAs, higher than in coffee beverages. Xanthones (mangiferin and isomangiferin), which haven't been found in other coffee parts, were found only in C. arabica and C. eugenioides and their young leaves showed high and comparable amounts to those found in the leaves of mango tree and honeybush shoots. In both species, the xanthones content was highly affected during leaf aging, as observed for 5-CQA and 3,5-DiCQA.

Recently, it was proved that tea made from the coffee leaves of $C$. arabica have strong antioxidant and anti-inflammatory properties, mainly due to the presence of phenolic compounds, in particular 5 -CQA and mangiferin, which are the two most abundant $[25,26]$. The present study suggests that the leaves of $C$. eugenioides are more promising than $C$. arabica leaves to produce coffee tea leaf, mainly due to their low economic relevance (low beans yield) and higher content in polyphenols, more specifically in mangiferin. Additionally, C. eugenioides leaves are able to produce a beverage which is naturally decaffeinated, reducing considerably the price for people who are sensitive to caffeine or wish to limit its intake. Therefore, further research on the potential benefits of a coffee leaf tea made from C. eugenioides leaves would be desirable.

The data overall confirm that indeed coffee leaf tea has the phytochemical profile to be used in the food industry as an alternative to tea, and that the choice of botanical species and their degree of maturity needs to be carefully evaluated, given their impact on the concentration of bioactive compounds.

Supplementary Materials: The following are available online at http://www.mdpi.com/2076-3921/9/1/6/s1, Table S1: Dimension of fresh leaves $(\mathrm{cm})$ of the different Coffea species at different development stages, Table S2: Electrospray ionization mass-spectrometry characterization of isomers, Table S3: Intra- and interday precision and accuracy of the HPLC standards of chlorogenic acids (CGAs), alkaloids and mangiferin.

Author Contributions: Conceptualization, S.C. and L.N.; methodology, Â.M. and S.C; software, Â.M. and S.C.; validation, Â.M.; formal analysis, Â.M.; investigation, Â.M.; resources, S.C., L.N., H.G.A., L.G.-G., M.D.C.S.; data curation, Â.M. and S.C.; writing-original draft preparation, Â.M., L.N., M.R.; writing-review and editing, S.C., L.N., H.G.A., L.G.-G., M.D.C.S., M.R.; supervision, S.C., L.N.; funding acquisition, M.R. and L.N. All authors have read and agreed to the published version of the manuscript.

Funding: This research was funded by H2020 Marie Skłodowska-Curie Actions, grant number 642014.

Acknowledgments: Paola Crisafulli and Lorenzo del Terra are acknowledged for technical assistance with sample preparations.

Conflicts of Interest: The authors declare no conflict of interest. The funders had no role in the design of the study; in the collection, analyses, or interpretation of data; in the writing of the manuscript, or in the decision to publish the results.

\section{References}

1. Abbas, M.; Saeed, F.; Anjum, F.M.; Afzaal, M.; Tufail, T.; Bashir, M.S.; Ishtiaq, A.; Hussain, S.; Suleria, H.A.R. Natural polyphenols: An overview. Int. J. Food Prop. 2017, 20, 1689-1699. [CrossRef] 
2. Han, X.Z.; Shen, T.; Lou, H.X. Dietary polyphenols and their biological significance. Int. J. Mol. Sci. 2007, 8, 950-988. [CrossRef]

3. Zhang, H.; Tsao, R. Dietary polyphenols, oxidative stress and antioxidant and anti-inflammatory effects. Curr. Opin. Food Sci. 2016, 8, 33-42. [CrossRef]

4. Perez-Jimenez, J.; Fezeu, L.; Touvier, M.; Arnault, N.; Manach, C.; Hercberg, S.; Galan, P.; Scalbert, A. Dietary intake of 337 polyphenols in French adults. Am. J. Clin. Nutr. 2011, 93, 1220-1228. [CrossRef] [PubMed]

5. Taguchi, C.; Fukushima, Y.; Kishimoto, Y.; Suzuki-sugihara, N.; Saita, E. Estimated dietary polyphenol intake and major food and beverage sources among elderly Japanese. Nutrients 2015, 7, 10269-10281. [CrossRef]

6. Ovaskainen, M.; Törrönen, R.; Koponen, J.M.; Sinkko, H.; Hellström, J.; Reinivuo, H.; Mattila, P. Dietary intake and major food sources of polyphenols in Finnish adults. J. Nutr. 2008, 138, 562-566. [CrossRef]

7. International Coffee Organization. Total Production by All Exporting Countries; International Coffee Organization: London, UK, 2018.

8. Ludwig, I.A.; Clifford, M.N.; Lean, M.E.J.; Ashihara, H.; Crozier, A. Coffee: Biochemistry and potential impact on health. Food Funtion 2014, 5, 1695-1717. [CrossRef]

9. Campa, C.; Petitvallet, A. Beneficial compounds from coffee leaves. In Achieving Sustainable Cultivation of Coffee; Lashermes, P., Ed.; Burleigh Dodds Science Publishing: Cambridge, UK, 2018.

10. Ashihara, H.; Monteiro, A.; Gillies, F.M.; Crozier, A. Biosynthesis of caffeine in leaves of coffee. Plant Physiol. 1996, 111, 747-753. [CrossRef]

11. Campa, C.; Mondolot, L.; Rakotondravao, A.; Bidel, L.P.; Gargadennec, A.; Couturon, E.; La Fisca, P.; Rakotomalala, J.; Jay-allemand, C.; Davis, A.P. A survey of mangiferin and hydroxycinnamic acid ester accumulation in coffee (Coffea) leaves: Biological implications and uses. Ann. Bot. 2012, 110, 595-613. [CrossRef]

12. Trevisan, M.T.S.; De Almeida, R.F.; Soto, G.; De Melo, E.; Filho, V.; Ulrich, C.M.; Owen, R.W. Quantitation by HPLC-UV of mangiferin and isomangiferin in coffee (Coffea arabica) leaves from Brazil and Costa Rica after solvent extraction and infusion. Food Anal. Methods 2016, 9, 2649-2655. [CrossRef]

13. de Almeida, R.F.; Trevisan, M.T.S.; Thomaziello, R.A.; Breuer, A.; Klika, K.D.; Ulrich, C.M.; Owen, R.W. Nutraceutical compounds: Echinoids, flavonoids, xanthones and caffeine identified and quantitated in the leaves of Coffea arabica trees from three regions of Brazil. Food Res. Int. 2019, 115, 493-503. [CrossRef] [PubMed]

14. Farah, A.; Donangelo, C.M. Phenolic compounds in coffee. Braz. J. Plant Physiol. 2006, 18, 23-36. [CrossRef]

15. Stalmach, A.; Steiling, H.; Williamson, G.; Crozier, A. Bioavailability of chlorogenic acids following acute ingestion of coffee by humans with an ileostomy. Arch. Biochem. Biophys. 2010, 501, 98-105. [CrossRef] [PubMed]

16. Mondolot, L.; La Fisca, P.; Buatois, B.; Talansier, E.; De Kochko, A.; Campa, C. Evolution in caffeoylquinic acid content and histolocalization during Coffea canephora leaf development. Ann. Bot. 2006, 98, 33-40. [CrossRef] [PubMed]

17. Campa, C.; Urban, L.; Mondolot, L.; Fabre, D.; Roques, S.; Lizzi, Y.; Aarrouf, J.; Doulbeau, S.; Breitler, J.; Letrez, C.; et al. Juvenile coffee leaves acclimated to low light are unable to cope with a moderate light increase. Front. Plant Sci. 2017, 8, 1-16. [CrossRef] [PubMed]

18. Pinto, M.; Sousa, M.; Nascimento, M. Xanthone Derivatives: New Insights in Biological Activities. Curr. Med. Chem. 2005, 12, 2517-2538. [CrossRef]

19. Joubert, E.; Gelderblom, W.C.A.; Louw, A.; de Beer, D. South African herbal teas: Aspalathus linearis, Cyclopia spp. and Athrixia phylicoides-A review. J. Ethnopharmacol. 2008, 119, 376-412. [CrossRef]

20. Barreto, J.C.; Trevisan, M.T.S.; Hull, W.E.; Erben, G.; De Brito, E.; Pfundstein, B.; Wurtele, G.; Spiegelhalder, B.; Owen, R.W. Characterization and quantitation of polyphenolic compounds in bark, kernel, leaves and peel of mango (Mangifera indica L.). J. Agric. Food Chem. 2008, 56, 5599-5610. [CrossRef]

21. Nehlig, A. Interindividual Differences in Caffeine Metabolism and Factors Driving Caffeine Consumption. Pharmacol. Rev. 2018, 70, 384-411. [CrossRef]

22. Allred, K.F.; Yackley, K.M.; Vanamala, J.; Allred, C.D. Trigonelline Is a Novel Phytoestrogen in Coffee Beans. J. Nutr. Biochem. Mol. Genet. Mech. 2009, 139, 1833-1838. [CrossRef] 
23. Azam, S.; Hadi, N.; Khan, N.U.; Hadi, S.M. Antioxidant and prooxidant properties of caffeine, theobromine and xanthine. Med. Sci. Monit. 2003, 9, BR325-BR330. [PubMed]

24. Tellone, E.; Galtieri, A.; Giardina, B.; Russo, A.; Bellocco, E.; Barreca, D.; Ficarra, S. Antioxidant Activity of Caffeine: A Focus on Human Red Blood Cells and Correlations with Several Neurodegenerative Disorders. In Coffee in Health and Disease Prevention; Elsevier Inc.: London, UK, 2015.

25. Chen, X.; Mu, K.; Kitts, D.D. Characterization of phytochemical mixtures with inflammatory modulation potential from coffee leaves processed by green and black tea processing methods. Food Chem. 2019, 271, 248-258. [CrossRef] [PubMed]

26. Chen, X.M.; Ma, Z.; Kitts, D.D. Effects of processing method and age of leaves on phytochemical profiles and bioactivity of coffee leaves. Food Chem. 2018, 249, 143-153. [CrossRef] [PubMed]

27. Clifford, M.N.; Kirkpatrick, J.; Kuhnert, N.; Roozendaal, H.; Salgado, P.R.; Kiikpatrick, J.; Kuhnert, N.; Roozendaal, H.; Salgado, P.R. LC-MSn analysis of the cis isomers of chlorogenic acids. Food Chem. 2008, 106, 379-385. [CrossRef]

28. Gumustas, M.; Kurbanoglu, S.; Uslu, B.; Ozkan, S.A. UPLC versus HPLC on Drug Analysis: Advantageous, Applications and Their Validation Parameters. Chromatographia 2013, 76, 1365-1427. [CrossRef]

29. Perrone, D.; Donangelo, C.M.; Farah, A. Fast simultaneous analysis of caffeine, trigonelline, nicotinic acid and sucrose in coffee by liquid chromatography-mass spectrometry. Food Chem. 2008, 110, 1030-1035. [CrossRef]

30. Jeon, J.; Kim, H.; Jeong, I.; Hong, S.; Oh, M.; Park, K.; Shim, J.; Abd El-Aty, A.M. Determination of chlorogenic acids and caffeine in homemade brewed coffee prepared under various conditions. J. Chromatogr. B 2017, 1064, 115-123. [CrossRef]

31. Clifford, M.N.; Johnston, K.; Knight, S.; Kuhnert, N. Hierarchical Scheme for LC-MS Identification of Chlorogenic Acids. J. Agric. Food Chem. 2003, 51, 2900-2911. [CrossRef]

32. Zheng, X.; Ashihara, H. Distribution, biosynthesis and function of purine and pyridine alkaloids in Coffea arabica seedlings. Plant Sci. 2004, 166, 807-813. [CrossRef]

33. Ky, C.; Louarn, J.; Dussert, S.; Guyot, B.; Hamon, S.; Noirot, M. Caffeine, trigonelline, chlorogenic acids and sucrose diversity in wild Coffea arabica L. and C. canephora P. accessions. Food Chem. 2001, 75, 223-230. [CrossRef]

34. Farah, A.; Monteiro, M.C.C.; Calado, V.; Franca, A.S.S.; Trugo, L.C.C. Correlation between cup quality and chemical attributes of Brazilian coffee. Food Chem. 2006, 98, 373-380. [CrossRef]

35. Nagai, C.; Rakotomalala, J.J.; Katahira, R.; Li, Y.; Yamagata, K.; Ashihara, H. Production of a new low-caffeine hybrid coffee and the biochemical mechanism of low caffeine accumulation. Euphytica 2008, 164, 133-142. [CrossRef]

36. Priolli, R.H.G.; Mazzafera, P.; Siqueira, W.J.; Möller, M.; Zucchi, M.I.; Ramos, L.C.S.; Gallo, P.B.; Colombo, C.A. Caffeine inheritance in interspecific hybrids of Coffea arabica $\times$ Coffea canephora (Gentianales, Rubiaceae). Genet. Mol. Biol. 2008, 31, 498-504. [CrossRef]

37. Cheng, B.; Furtado, A.; Smyth, H.E.; Henry, R.J. Influence of genotype and environment on coffee quality. Trends Food Sci. Technol. 2016, 57, 20-30. [CrossRef]

38. Ashihara, H.; Sano, H.; Crozier, A. Caffeine and related purine alkaloids: Biosynthesis, catabolism, function and genetic engineering. Phytochemistry 2008, 69, 841-856. [CrossRef] [PubMed]

39. Magalhães, S.T.V.; Guedes, R.N.C.; Demuner, A.J.; Lima, E.R. Effect of coffee alkaloids and phenolics on egg-laying by the coffee leaf miner Leucoptera coffeella. Bull. Entomol. Res. 2008, 98, 483-489. [CrossRef]

40. Oestreich-Janzen, S. Chemistry of Coffee. In Comprehensive Natural Products II Chemistry and Biology; Elsevier: Kidlington, UK, 2010.

41. Koshiro, Y.; Jackson, M.C.; Katahira, R.; Wang, M.L.; Nagai, C.; Ashihara, H. Biosynthesis of chlorogenic acids in growing and ripening fruits of Coffea arabica and Coffea canephora plants. Zeitschrift für Naturforschung $C$ 2007, 62, 731-742. [CrossRef] [PubMed]

42. Campa, C.; Doulbeau, S.; Dussert, S.; Hamon, S.; Noirot, M. Qualitative relationship between caffeine and chlorogenic acid contents among wild Coffea species. Food Chem. 2005, 93, 135-139. [CrossRef]

43. Shang, X.; Pan, H.; Li, M.; Miao, X.; Ding, H. Lonicera japonica Thunb.: Ethnopharmacology, phytochemistry and pharmacology of an important traditional Chinese medicine. J. Ethnopharmacol. 2011, 138, 1-21. [CrossRef] 
44. The Commission of the PPRC. Pharmacopoeia of People's Republic of China; China Medical Science and Technology Press: Beijing, China, 2015.

45. Imran, M.; Arshad, M.S.; Butt, M.S.; Kwon, J.H.; Arshad, M.U.; Sultan, M.T. Mangiferin: A natural miracle bioactive compound against lifestyle related disorders. Lipids Health Dis. 2017, 16, 1-17. [CrossRef]

(C) 2019 by the authors. Licensee MDPI, Basel, Switzerland. This article is an open access article distributed under the terms and conditions of the Creative Commons Attribution (CC BY) license (http://creativecommons.org/licenses/by/4.0/). 